\title{
APLICACIÓN DE LOS AJUSTES RAZONABLES EN QATAR. UN ANÁLISIS SOBRE LA GARANTÍA DE LA IGUALDAD DE LAS PERSONAS CON DISCAPACIDAD EN EL DERECHO COMÚN QATARÍ ${ }^{\delta}$
}

\author{
APPLICATION OF REASONABLE ACCOMMODATION IN QATAR. AN \\ ANALYSIS ON THE GUARANTEE OF THE EQUALITY OF PERSONS WITH \\ DISABILITIES IN THE QATARI LAW
}

\author{
Rafael De Asís Roig*, M. Carmen Barranco Avilés**, María Laura \\ Serra***, Patricia Cuenca Gómez', Francisco Javier Ansuátegui \\ Roig**, Khalid Al Ali ${ }^{\gamma}$ y Pablo Rodríguez del Pozo ${ }^{\gamma /}$
}

\begin{abstract}
RESUMEN: Este trabajo considera la conceptualización y aplicación de la figura de los ajustes razonables en Qatar tras nueve años desde la ratificación de la Convención sobre los Derechos de las Personas con Discapacidad (CDPD). En él se trata de analizar la situación de igualdad y no discriminación de las personas con discapacidad utilizando como medida de impacto la figura de ajustes razonables. El artículo destaca las principales fallas y virtudes del Estado de Qatar respecto a esta figura y traza un camino a fin de poder avanzar en la inclusión de las personas con discapacidad a la luz de la CDPD.
\end{abstract}

\begin{abstract}
This paper considers the conceptualization and application of reasonable acommodation in Qatar after nine years since the ratification of the Convention on the Rights of Persons with Disabilities (CRPD). It analyzes the situation of equality and nondiscrimination of people with disabilities using the figure of reasonable adjustments as an impact measure. The article highlights the main failures and virtues of the State of Qatar regarding reasonable accommodation and draws a path to move forward in the inclusion of people with disabilities in the light of the CRPD.
\end{abstract}

PALABRAS CLAVE: ajustes razonables, igualdad, CDPD, Qatar.

KEYWORDS: reasonable accommodation, equality, CRPD, Qatar.

Fecha de recepción: 07/11/2017

Fecha de aceptación: 21/11/2017

doi: https://doi.org/10.20318/universitas.2018.4020

$\delta$ Esta investigación fue posible gracias al subsidio NPRP-7-380-5-051 de Qatar National Research Fund (miembro de Qatar Foundation). Las afirmaciones hechas en este documento son únicamente responsabilidad de los/as autores/as. [This publication was made possible by the NPRP award NPRP-7-380-5-051 from the Qatar National Research Fund (a member of The Qatar Foundation). The statements made herein are solely the responsibility of the authors].

* Catedrático de Filosofía del Derecho en la Universidad Carlos III de Madrid. Email: rafael.asis@uc3m.es

** Profesora Titular de Filosofía del Derecho en la Universidad Carlos III de Madrid. E-mail: mcarmen.barranco@uc3m.es

*** Investigadora Asociada en el Centre for Disability Law and Policy de la Universidad Nacional de Irlanda en Galway. E-mail: marialaura.serra@nuigalway.ie

- Profesora Visitante en la Universidad Carlos III de Madrid. E-mail: patricia.cuenca@uc3m.es

- Catedrático de Filosofía del Derecho en la Universidad Carlos III de Madrid. Email: javofil@der-pu.uc3m.es

${ }^{\gamma}$ Professor of Health Sciences, Qatar University. E-mail: kalali@qu.edu.qa

${ }^{r}$ Associate Professor of Medical Ethics in Medicine, Joan and Sanford I. Weill Cornell

Medical College in Qatar. E-mail: prd2002@qatar-med.cornell.edu 


\section{I.- INTRODUCCION}

La garantía de la protección y promoción de los derechos humanos para las personas con discapacidad es más inestable que para otras poblaciones de personas. Esta situación se agrava si estas personas se encuentran en zonas y/o situaciones de emergencia o de desastres naturales. Que la situación discriminatoria sea más agravante se debe a la existencia de ciertos prejuicios y estereotipos que producen una desigualdad en sus oportunidades de vida, tanto en el ámbito público como privado.

La Convención sobre los Derechos de las Personas con Discapacidad (CDPD) viene a deshacer esos prejuicios y a luchar contra la desigualdad desde un aspecto jurídico. Pero la CDPD no es únicamente una lucha contra la discriminación, sino que "mezcla ideas acerca de la igualdad y no discriminación con una red de derechos sustantivos" ${ }^{1}$. La CDPD en definitiva, es una herramienta para la normalización de la sociedad a través de la lucha contra el discurso de lo especial que tradicionalmente ha estado asociado a la discapacidad, y a través de la exaltación de la riqueza que la diversidad produce en la sociedad.

En este aspecto, resulta lógico pensar que la CDPD, siendo un instrumento de derechos humanos, se constituya en una herramienta de lucha contra la discriminación. Y es que la historia de los derechos humanos puede ser representada precisamente como un proceso contra la discriminación.

En términos generales, la discriminación se produce, bien cuando diferencias no relevantes son tenidas en cuenta para producir directa o indirectamente un trato diferente o bien cuando diferencias relevantes no son tenidas en cuenta para producir directa 0 indirectamente un trato diferente ${ }^{2}$.

Tradicionalmente la lucha contra la discriminación se ha desarrollado a través de dos estrategias que Rafael de Asís Roig denomina 'estrategia de la situación' y 'estrategia de la identidad'3.

La estrategia de la situación centra su lucha contra la discriminación en la situación en la que se encuentran ciertas

\footnotetext{
${ }^{1}$ QUINN, G. y DOYLE, S., Getting a Life - Living Independently and Being Included in the Community, Office of the High Commissioner for Human Rights - Regional Office for Europe, Brussels, 2012, p. 16. Disponible en:

http://europe.ohchr.org/Documents/Publications/Getting_a_Life.pdf (última consulta: 30 de mayo de 2017)

2 Vid. CUENCA GÓMEZ, P. y DE ASÍS, R., "La igualdad de oportunidades de las personas con discapacidad", en Pérez Bueno, L.C. y Álvarez Ramírez, G., 20032012: 10 años de legislación sobre no discriminación de personas con discapacidad en España. Estudios en homenaje a Miguel Ángel Cabra de Luna, Fundación Derecho y Discapacidad, Madrid, 2012, pp. 53 y ss.

${ }^{3}$ Vid. DE ASÍS, R., "Las situaciones de dependencia desde un enfoque de derechos humanos", en Ramiro, M.A. y Cuenca, P. (ed.), Los derechos humanos: la utopía de los excluidos, Dykinson, Madrid, 2010, pp. 163 y ss.
} 
personas a las que bien se las trata de manera igual, bien de manera diferente de forma injustificada. Se busca así corregir una discriminación y para ello no se apela a la persona o grupo discriminado sino a una situación. En este sentido, la estrategia de la situación se desenvuelve dentro del proceso de generalización de los derechos ya que normalmente la situación de discriminación se produce porque hay insatisfacción de derechos que otros si tienen satisfechos. Y la idea de igualdad que se utiliza es la de la igualdad como punto de partida y la igualdad como generalidad (con independencia de que, con carácter temporal y ciertamente especial, se justifiquen medidas de diferenciación positiva $)^{4}$.

Por su parte, la estrategia de la identidad centra su lucha contra la discriminación en los rasgos, en la identidad, de una persona o grupo a la o a los que se trata bien de manera igual, bien de manera diferenciada de forma injustificada. Se busca corregir una discriminación apelando a la persona o al grupo. Así, la estrategia de la identidad se desenvuelve dentro del proceso de especificación de los derechos, demandando el reconocimiento de derechos a personas o colectivos concretos.

Ambas estrategias están presentes en la CDPD, la cual, en su Artículo 5.2 establece: "Los Estados Parte prohibirán toda discriminación por motivos de discapacidad y garantizarán a todas las personas con discapacidad protección legal igual y efectiva contra la discriminación por cualquier motivo."

En este sentido, la CDPD se basa en la apuesta por una sociedad inclusiva, en la que la diversidad ocupa un lugar central. Tal apuesta por una sociedad inclusiva, por una sociedad que se adapta al pluralismo, es una consecuencia de un enfoque de derechos, ya que este enfoque está irremediablemente unido a la inclusión. Y también lo es del modelo social, cuya concepción de la discapacidad se decanta por la inclusión frente a la integración.

Para ciertos grupos en situación de vulnerabilidad, la CDPD utiliza el llamado 'enfoque de la doble vía' para la protección de derechos humanos. Estos grupos son el de los niños y niñas en su Artículo 7, las mujeres en su Artículo 6 y también se puede interpretar que utiliza este enfoque de la doble vía para todas las personas con discapacidad cuando se presentan situaciones de riesgo, incluidas situaciones de conflicto armado, emergencias humanitarias y desastres naturales (Artículo 11). El enfoque de la doble vía se refiere a garantizar los derechos humanos a todas las personas de manera general, aportando al mismo tiempo visibilidad a su situación en un artículo específico.

La CDPD reconoce que estos grupos se encuentran sujetos a diferentes formas de discriminación. Expresamente lo establece para las mujeres con discapacidad en su Artículo 6, siendo el único y el primer tratado de derechos humanos que hace una referencia

\footnotetext{
${ }^{4}$ Ibídem.
} 
explícita a la discriminación múltiple de las mujeres y niñas con discapacidad. En este sentido, los Estados parte de la CDPD -entre ellos el Estado de Qatar- deben de "tomar medidas pertinentes para asegurar el pleno desarrollo, adelanto y potenciación de la mujer, con el propósito de garantizarle el ejercicio y goce de los derechos humanos y las libertades fundamentales establecidos en la presente Convención." 5

En la CDPD, como derecho, la igualdad y no discriminación viene acompañando de ciertos principios establecidos en la CDPD (en su Artículo 3), que representan una asistencia a fines de interpretar e implementar la Convención. En igual sentido, los derechos y libertades de las personas con discapacidad incluidos en la CDPD así como las obligaciones de los Estados parte derivan de estos principios. Es decir, el texto del Artículo 5 en sí mismo representa una base respecto del mandato de los Estados parte en lo que tiene que ver con tomar medidas al respecto y asegurar todos los derechos humanos y libertades fundamentales a las personas con discapacidad. Pero ese mandato parte del reconocimiento de que a las personas con discapacidad debe respetárseles su dignidad inherente y autonomía individual (Artículo 3, CDPD).

Una herramienta en pos de la igualdad y no discriminación que la CDPD contiene como novedosa en materia de discapacidad y de la que pretende hacerse eco este artículo, es la de los ajustes razonables. Los ajustes razonables son definidos en la CDPD (Artículo 3) como las "modificaciones y adaptaciones necesarias y adecuadas que no impongan una carga desproporcionada o indebida, cuando se requieran en un caso particular, para garantizar a las personas con discapacidad el goce o ejercicio, en igualdad de condiciones con las demás, de todos los derechos humanos y libertades fundamentales".

Los ajustes razonables son medidas que pretenden adaptar el entorno, bienes y servicios a las específicas necesidades de una persona, asegurando de esta manera la inclusión de ellas en la sociedad y por lo tanto garantizando su derecho a la igualdad. Mediante los ajustes razonables se pueden garantizar los derechos, ya que en ocasiones se trata de una precondición al ejercicio de los mismos. De Asís Roig explica que el ajuste razonable es uno de los ejes sobre los que gira el reconocimiento de los derechos de las personas con discapacidad ${ }^{6}$. Pero la naturaleza de este deber (el de proporcionar ajustes razonables), al decir de Lawson, "varía ligeramente en diferentes contextos $(\ldots)^{\prime \prime}$. De este modo, al variar los

\footnotetext{
${ }^{5}$ Artículo 6, CDPD.

6 Vid. DE ASÍS ROIG, R., "Lo razonable en el concepto de ajuste razonable", en Salmón E. y Bregalio R. (eds.), Nueve conceptos claves para entender la Convención sobre los derechos de las personas con discapacidad, Pontificia Universidad Católica del Perú, Lima, 2015, pp. 99 y ss.

7 LAWSON, A., "Challenging Disabling Barriers to Information and Communication Technology in the Information Society : A United Kingdom Perspective", en
} 
contextos también varían los ajustes razonables (como por ejemplo ocurre en el ámbito de la participación política). Pero además, los ajustes razonables pueden variar también en cuanto al contexto del Estado del que estemos hablando.

La investigación jurídica respecto de la igualdad y no discriminación, implica referirse no sólo a los derechos civiles y políticos, sino también a los derechos económicos, sociales y culturales. Al respecto, el marco jurídico del Estado de Qatar complejiza un tanto la situación a la que hace referencia este artículo pese a que en el plano regional Qatar ha ratificado la Carta Árabe de Derechos Humanos, la cual contiene el derecho a la igualdad y no discriminación ante la ley (Artículo 11); el reconocimiento de todas las personas ante la ley (Artículo 22) y la igualdad ante las Cortes y Tribunales (Artículo 22), entre otros derechos.

El artículo se divide en cuatro secciones y luego de la introducción, la segunda sección nos sitúa en las conceptualizaciones necesarias a fin de entender el fondo intelectual con el que este artículo trabaja. Seguidamente, la siguiente sección nos pone en contexto, describiendo la situación jurídica y social de Qatar. Es necesario comprender que estamos escribiendo acerca de un contexto jurídico diferente al que se acostumbra en Occidente puesto que Qatar se destaca por tener un derecho común y un derecho islámico. Esta división en ciertos derechos juega un rol fundamental.

La cuarta sección traza ciertos pasos que como autores pensamos que beneficiarían al Estado y a la sociedad de Qatar en pos de una real inclusión de las personas con discapacidad que habitan su suelo. En este sentido y habiendo considerado las observaciones que organismos internacionales le han realizado a Qatar, tratamos de configurar una hoja de ruta bajo la luz tanto de la Convención sobre los Derechos de las Personas con Discapacidad como del modelo social de discapacidad.

La última sección por su parte se refiere a una conclusión del trabajo y repasar los puntos clave que Qatar debería de tener en cuenta para una aplicación de los ajustes razonables.

\section{II.- CONTEXTO JURÍDICO-SOCIAL DE QATAR}

Qatar es un Estado Árabe soberano e independiente emplazado en la costa occidental del Golfo Pérsico. Allí, convive una sociedad tradicionalmente musulmana junto a una sociedad de personas expatriadas de distintas regiones del mundo, en su mayoría provenientes de la India y Filipinas, cuyas religiones son variadas. En el plano normativo, Qatar responde a un dualismo jurídico. Así, a partir de su independencia (1971) del imperio británico gran parte de

Waddington L. y Quinn G. (eds.), European Yearbook of Disability Law - Volume 2, Intersentia, Mortsel, 2010, p. 136. 
su jurisdicción corresponde al derecho continental (Adlia court) ${ }^{8}$, sin embargo la Sharia islámica se aplica en varios aspectos, sobre todo el derecho de familia. A pesar de que en la nueva Constitución del año $2005^{9}$ se ven indicios de una sociedad más liberal dentro de lo que son los Estados árabes, "la ley de la Sharia es reconocida en la Constitución como la principal fuente de legislación (Artículo 1)"10.

Así, la Constitución Permanente del Estado de Qatar, adoptada el 8 de junio de 2004, establece como religión oficial el Islam, a la Sharia islámica como fuente principal de legislación y a la democracia como sistema político. Asimismo establece que el pueblo de Qatar es parte de la Nación Árabe y su lengua oficial es el árabe. El gobierno del Estado de Qatar se hereda en la familia de Al-Thani y solo por línea de descendencia masculina de Hamad Bin Khalifa Bin Hamad Bin Abdullah Bin Jassim. El jefe del Estado es el Emir, en quien el poder ejecutivo es investido y quien organiza el Consejo de Ministros. La Autoridad Legislativa está a cargo del Consejo de Al-Shoura, un órgano consultivo compuesto por cuarenta y cinco miembros, treinta de los cuales son elegidos en una votación general secreta y directa, y los quince restantes son nombrados por el Emir. ${ }^{11}$

\section{II.1.- Qatar y las personas con discapacidad. A nueve años de la ratificación de la CDPD.}

El Estado de Qatar ha firmado la CDPD en el año 2007, ratificándola al año siguiente. Respecto a su Protocolo, Qatar lo ha firmado en el año 2007 pero al día de hoy sigue sin ser ratificado. El Protocolo Facultativo de la CDPD otorga al Comité sobre los Derechos de las Personas con Discapacidad (desde ahora en más, Comité de la CDPD) la competencia para examinar las denuncias individuales relativas a presuntas violaciones de la CDPD por los Estados Parte en el Protocolo.

Pese a no haber ratificado el Protocolo, que el Estado de Qatar haya ratificado la CDPD es una muestra de una intención de avanzar en los derechos de las personas con discapacidad. Así como para Qatar, para todos los Estados que han mantenido hasta ahora una

\footnotetext{
8 Para saber acerca del dualismo jurídico de Qatar, véase por ej.: HAMZEH, A., Qatar: the duality of the legal system. Middle Eastern Studies, 30(1), 1994, pp.7990.

9 La Constitución Permanente del Estado de Qatar fue ratificada mediante un referendo público el 29 de abril de 2003, promulgada por el Emir de Qatar el 8 de junio de 2004 y entró en vigencia el 9 de junio de 2005. En ella se reconoce el principio de división de poderes en su artículo 60. Consejo de Derechos Humanos, Informe de la Relatora Especial sobre la independencia de jueces, juezas, abogados y abogadas, Gabriela Knaul, en su misión a Qatar. A/HRC/29/26/Add.1, párr. 6.

${ }_{10}$ A/HRC/29/26/Add.1, párr. 9.

${ }^{11}$ MINISTRY OF FOREIGN AFFAIRS, Permanent Constitution of the State of Qatar, 2008. Disponible en:

https://web.archive.org/web/20041024231923/http://english.mofa.gov.qa/details. cfm?id=80 (última consulta: 30 de mayo de 2017).
} 
fidelidad al modelo médico, adecuar las políticas y legislaciones relativas a los derechos de las personas con discapacidad, no es un proceso fácil, no por razones económicas o políticas, sino porque el proceso implica ciertos cambios de mentalidades muy arraigadas no solo en la sociedad sino en el Derecho en sí mismo.

En el marco del proyecto de investigación The impact of the International Convention on the Rights of Persons with Disabilities on the internal legislation of Qatar: Analysis and proposals, se organizaron en la ciudad de Doha en Qatar, reuniones y visitas a Hospitales, Escuelas, Universidades, Instituciones públicas y privadas dedicadas especialmente a las personas con discapacidad, a la Comisión de Derechos Humanos de Qatar, Departamentos de Gobierno dedicados, entre otros temas, a la discapacidad, etc. En tales ocasiones se pudo observar que es indudable que el Estado de Qatar ha destinado fondos e invertido, sobre todo en tecnología, para las personas con discapacidad. Esto da muestras de una cierta evolución gradual desde el modelo médico al modelo social de la discapacidad en vistas a cumplir con las aspiraciones de una estrategia nacional denominada Qatar National Vision 2030 (QNV 2030), en materia de justicia social e inclusión en la que, desde el año 2008, el Estado de Qatar está involucrado. De acuerdo a su plataforma, QNV 2030 busca convertir a Qatar en un país vibrante y próspero en el que haya justicia económica y social, en armonía con la naturaleza ${ }^{12}$. Gran parte de la responsabilidad de los avances hacia un modelo social de la discapacidad lo tiene el Comité Nacional de Derechos Humanos del Estado de Qatar quien pidió al Gobierno que agilice los procedimientos para la aplicación de las enmiendas propuestas a la Ley Número 2 de 2004 (referida a personas con necesidades especiales). En el año 2012 el Consejo de Ministros de Qatar aprobó el proyecto de resolución que la modifica. ${ }^{13}$

Pese a esto, la manera en que es concebido el trato y tratamiento a las personas con discapacidad parte de la legislación. Y la legislación en Qatar se encuentra principalmente dentro del alcance del modelo médico. Se trata a las personas con discapacidad como personas enfermas a ser rehabilitadas, pero no incluidas en la sociedad. Muestra de esto es el énfasis que pone el Estado en la salud respecto de las políticas para las personas con discapacidad. No hay un trato transversal de la discapacidad, sino que más bien la

12 GENERAL SECRETARIAT FOR DEVELOPMENT PLANNING, Qatar National Vision 2030, General Secretariat for Development Planning, Doha, 2008. Disponible en:

http://www.mdps.gov.qa/en/qnv/Documents/QNV2030_English_v2.pdf (última consulta: 30 de mayo de 2017).

13 Véase: MINISTRY OF DEVELOPMENT PLANING AND STATISTICS, Qatar's Fourth National Human Developent Report - Realising Qatar National Vision 2030: The Right to Develpment, Ministry of Development Planning and Statistics, Doha, 2015. Disponible en:

http://hdr.undp.org/sites/default/files/qatar_nhdr4_english_15june2015.pdf (última consulta: 30 de mayo de 2017). 
discapacidad se encuentra sectorizada en el ámbito de la salud y sin una perspectiva de derechos humanos.

Se puede ejemplificar esta afirmación, con la definición de las personas con 'necesidades especiales' en el Artículo 1 de la Ley Número 2 de 2004, en donde son definidas como "cualquier persona con una discapacidad permanente, parcial o total, en cualquiera de sus sentidos, o en su capacidad física, o en su capacidad psicológica o mental, a tal punto que su oportunidad de aprender o de someterse a rehabilitación o de ganarse la vida se vea limitada". Esta perspectiva también está presente en otras referencias con las que trabaja Qatar, tales como, "educación especial", "rehabilitación" e "Institutos de educación especial". Se entiende entonces que la discapacidad en Qatar es concebida como una situación médica ya que se entiende que la persona tiene necesidades 'especiales', siendo en gran parte el objetivo de la intervención el rehabilitar a las personas con discapacidad y no incluirlas desde una perspectiva inclusiva y desde una mirada de la sociedad tal y como se entiende a la discapacidad desde el modelo social y desde un enfoque de derechos humanos a través del articulado de la CDPD.

A pesar de que la CDPD no define a la discapacidad como tal, en su artículo primero expresa que cuando se habla de personas con discapacidad se están incluyendo a aquellas personas que tengan deficiencias físicas, mentales, intelectuales o sensoriales a largo plazo que, al interactuar con diversas barreras, puedan impedir su participación plena y efectiva en la sociedad, en igualdad de condiciones con las demás. En este mismo artículo, la CDPD establece su propósito, que se basa en promover, proteger y asegurar el goce pleno y en condiciones de igualdad de todos los derechos humanos y libertades fundamentales por todas las personas con discapacidad, y promover el respeto de su dignidad inherente. ${ }^{14}$

Al repasar el derecho común qatarí, uno de los principales problemas respecto a las personas con discapacidad, es más bien estructural, del cual su base conceptual se hace sentir. Por un lado, a pesar de los esfuerzos y del dinero que el Estado ha invertido para la población con discapacidad, tal como la estrategia nacional de desarrollo y de salud, Shafallah Center; instituciones educativas con alto nivel tecnológico para las personas con discapacidad sensorial, entre otras medidas (que responden a servicios más que a derechos), su filosofía radica -aunque no completamente- en el modelo médico/rehabilitador y no en el modelo social de la discapacidad. De manera general, el primero refiere a la discapacidad como un rasgo de la persona. En este modelo, se entiende que la persona con discapacidad es quien tiene que adaptarse al medio y no el medio a la persona. El modelo social de la discapacidad, por su parte, se refiere a la discapacidad como una construcción social. La discapacidad bajo este modelo se define como una situación de la persona y no como

${ }^{14}$ Artículo 1, CDPD. 
un rasgo de ella. Son las barreras del entorno (físicas, sensoriales, intelectuales $\mathrm{y} / \mathrm{o}$ del ambiente) las que discapacitan a la persona. Este modelo, el cual se ve claramente reflejado en el recién mencionado Artículo 1 de la CDPD así como en el resto de su articulado, ha inspirado la negociación y posterior firma de la Convención que se ha formulado bajo un modelo de derechos, el cual puede denominarse modelo de derechos humanos puesto que la CDPD es un Tratado de Derechos Humanos.

Entre las representaciones jurídicas mencionadas que dejan entrever que Qatar predominantemente incorpora el modelo médico/rehabilitador, también se encuentra el Código Civil qatarí y la ya mencionada Constitución Permanente del Estado de Qatar, en donde ya sea por no haber sido adecuados (reformados) con posterioridad a la ratificación de la CDPD por el Estado de Qatar, o por un fin protector, prevalece un enfoque de sustitución de la capacidad jurídica de las personas con discapacidad (es decir, no se legisla respetando el Artículo 12 de la CDPD), el Código Civil de Qatar niega el acceso a la justicia de las personas con discapacidad (referido en el Artículo 13 de la CDPD), por ejemplo, Artículos 71; 81; $85 ; 87 ; 157 ; 161 ; 372 ; 458$, entre otros $y$, además en la Constitución Nacional se interpreta a la discapacidad análoga a la incapacidad (por ejemplo, Artículos 15 y 101). En adhesión a ello, en Ley No. 25 de 2001 se puede ver claramente que se realiza un tratamiento de la discapacidad únicamente desde un aspecto de la salud (Artículo 8). Lo mismo pasa con la Ley No. 23 de 2004 respecto a la promulgación del Código de Procedimiento Penal, en donde predomina un modelo sustitutivo y en consecuencia una denegación del acceso a la justicia a las personas con discapacidad -'mental', según la ley- (Artículo 5), además de que se apela a la protección de la persona y no al reconocimiento como sujeto de derecho (Artículo 20); se suma además la sección 7ma de esta misma ley, relativa a las sentencias la cual está elaborada desde una perspectiva del modelo médico, identificándose a la discapacidad como una condición de la persona además de que se permite realiza un test de discapacidad.

Como ponen de relieve algunas de las consideraciones realizadas por el Comité de la CDPD en las Observaciones finales ${ }^{15}$, Qatar maneja todavía una concepción de la discapacidad que responde en lo esencial a la perspectiva asistencialista y al modelo medico alejada de una aplicación comprensiva y coherente del enfoque de derechos humanos y el modelo social. Bajo este aspecto, Qatar continúa rigiéndose por una legislación doméstica que se

${ }^{15}$ Comité sobre los Derechos de las Personas con Discapacidad, Observaciones finales sobre el informe inicial de Qatar, 2 de octubre de 2015. CRPD/C/QAT/CO/1, párr. 7. Vid. un análisis general de los desafíos que tiene qe afrontar Qatar en la implementación de la CDPD en De ASÍS ROIG, R.; BARRANCO AVILÉS, M.C.; CUENCA GÓMEZ, P.; RODRÍGUEZ DEL POZO, P.; AL ALI, K., "The Impact of the International Convention on the Rights of Persons with Disabilities on Qatari Domestic Legislation", The Age of Human Rights Journal, nro. 8, 2017. 
identifica con el modelo médico/rehabilitador, pese haber ratificado la CDPD. Esto no justifica el hecho de que Qatar como Estado parte no haya actualizado su legislación a la luz de la CDPD, pero deja una conciencia jurídico-política de que el proceso es lento y que en un Estado de la región de Arabia cuya legislación contiene dos tipos de derechos (continental y Sharia) el proceso se hace más lento y dificultoso aún.

\section{III.- CÓMO AVANZAR}

Decíamos que en este trabajo se analiza la igualdad y no discriminación de las personas con discapacidad en Qatar desde el aspecto de la figura de los ajustes razonables y desde un único aspecto jurídico con el fin de avanzar hacia una legislación inclusiva a la luz de la CDPD como Tratado de Derechos Humanos que Qatar ha ratificado. Vale decir que el análisis es en cierta medida parcial ya que se hace únicamente desde el llamado derecho común de Qatar.

Para abordar el análisis, las observaciones finales que el Comité de la CDPD ha realizado al Estado de Qatar consideramos son de extrema relevancia. Las Observaciones finales, son observaciones que el Comité de la CDPD realiza al Estado parte respecto al informe que éste presenta, y en cuyo texto aborda todos los artículos de la CDPD. Estas observaciones resaltan tanto aspectos positivos como factores y dificultades que obstaculizan la aplicación de la CDPD en dicho Estado parte. Asimismo, se resaltan los principales motivos de preocupación, y se realizan sugerencias y recomendaciones concretas sobre acciones futuras. ${ }^{16}$

Tomando como punto de partida las Observaciones finales del Comité de la CDPD, en los siguientes dos apartados se analiza el tratamiento de la discapacidad en el Estado de Qatar y el derecho a la igualdad y no discriminación, para luego analizar de manera más pormenorizada la figura de los ajustes razonables y cómo éstos son aplicados en el Estado de Qatar. En tal sentido, el análisis parte de la concepción de que existe un vacío legal en el derecho continental qatarí.

Como se menciona al comienzo de este artículo, el derecho a la igualdad es esencial para proteger y para ejercer los derechos humanos. Además de la CDPD, la Declaración Universal de Derechos Humanos, el Pacto Internacional de Derechos Civiles y Políticos, el Pacto Internacional de Derechos Económicos, Sociales y Culturales, la Convención sobre los Derechos del Niño (CDN), la Convención Internacional sobre la Eliminación de todas las Formas de Discriminación Racial (CERD) y la Convención sobre la Eliminación de todas las formas de Discriminación contra la Mujer (CEDAW, en sus

\footnotetext{
${ }^{16}$ Arts. 13 y 14 de los Métodos de trabajo del Comité sobre los Derechos de las Personas con Discapacidad adoptados en su quinto período de sesiones, publicado el 2 de septiembre de 2011, CRPD /C/5/4.
} 
siglas en inglés), especifican y garantizan el derecho a la igualdad y además conforman parte del marco normativo a aplicar -dentro del sistema de derecho internacional de los derechos humanos- en lo que se refiere a la situación de igualdad y no discriminación de las personas con discapacidad, aunque en el caso de la CEDAW, la CDN y la CERD sólo sean de aplicación para los grupos sociales referenciados en tales Convenciones. De los Tratados mencionados, el Estado de Qatar sólo ha ratificado la CEDAW (sin haber firmado ni ratificado su Protocolo) y la CDPD (respecto de la cual, como hemos señalado arriba ha firmado, pero no ratificado su Protocolo). Además, Qatar ha ratificado la Convención Internacional sobre la Eliminación de todas las Formas de Discriminación Racial, la Convención sobre los Derechos del Niño (y dos de sus tres Protocolos) y la Convención contra la Tortura y otros Tratos o Penas Crueles, Inhumanos o Degradantes.

En el plano doméstico, Qatar en su informe inicial al Comité de la CDPD esgrime que la igualdad es uno de los pilares sobre los que se erige la sociedad qatarí. ${ }^{17}$

El principio de igualdad, junto a otros principios, se consolida en el Artículo 19 de su Constitución cuando dice "El Estado protege los pilares de la sociedad y vela por la seguridad, la estabilidad y la igualdad de oportunidades para la ciudadanía." En dicho informe, Qatar afirma que todas las políticas del Estado deben incorporar la salvaguardia de los pilares de la sociedad consagradas en el Artículo 18 (cuyo texto dispone que la sociedad de Qatar se funda en los principios de justicia, caridad, libertad, igualdad y moralidad), incluido el principio de igualdad, por lo cual no pueden ser vulnerados por ninguna ley o norma de carácter legislativo ya que son un bien constitucional protegido.

Qatar explica que para reforzar y potenciar esta protección constitucional se estableció la Ley No 12 del 2008 en donde es el Alto Tribunal Constitucional el órgano jurisdiccional competente para resolver los recursos de inconstitucionalidad de las leyes y los reglamentos.

La Constitución qatarí también hace referencia a la igualdad ante la ley de toda la ciudadanía y específicamente afirma que no debe de haber discriminación en razón de sexo, origen, lengua o religión'. ${ }^{18}$ Asimismo, Qatar en dicho informe explica que "el derecho a la igualdad y a la no discriminación consagrado en la Constitución gobierna todas las instituciones y todos los organismos del Estado, que están obligados a hacer efectivos tales derechos y a evitar actos

${ }^{17}$ Informe Inicial presentado por el Estado de Qatar en virtud del artículo 35 de la CDPD, Qatar, 9 de julio de 2014, CRPD /C/QAT/1.

18 Arts. 34 y 35 del Título III de la Constitución Permanente del Estado de Qatar. Véase: MINISTRY OF FOREIGN AFFAIRS, Permanent Constitution of the State of Qatar, 2008. Disponible en:

https://web.archive.org/web/20041024231923/http://english.mofa.gov.qa/details. cfm?id=80 (última consulta: 30 de mayo de 2017). 
y prácticas discriminatorias o que inciten a la discriminación, con independencia de cuál sea la instancia que incurra en tales actos o prácticas. El ordenamiento constitucional y jurídico obliga al Estado y a todas sus instituciones a respetar los principios de igualdad, justicia y no discriminación." ${ }^{19}$

Recordando que la discriminación no solo se presenta en la ley sino también en la práctica y con todo este marco jurídico internacional y nacional de los derechos humanos, es necesario distinguir que la realidad jurídica qatarí hace que se torne un poco más difícil hablar de igualdad y no discriminación en la práctica de las personas con discapacidad, puesto que el Comité del ECOSOC, sobre todo -Pacto no ratificado por Qatar- ha conceptualizado a través de observaciones generales la igualdad y no discriminación en la práctica y lo ha hecho con más énfasis en el plano de las mujeres, niños y niñas con discapacidad, quienes tienen una larga historia confrontando discriminaciones y opresiones. ${ }^{20}$ Un punto a destacar es el hecho de que no tener ratificado el Protocolo de la CEDAW complica la garantía de protección y promoción de los derechos de las mujeres (con y sin discapacidad), ya que el Protocolo Facultativo de la CEDAW, entre otras funciones, establece los mecanismos de denuncia e investigación de la propia CEDAW, a través de su Comité. ${ }^{21}$ No obstante, hay que decir que Qatar no es el único Estado -incluyendo los Estados de Occidente- que no tenga ratificados ciertos Tratados de Derechos Humanos y/o Protocolos Facultativos pertenecientes a los Tratados, sin embargo el problema se encuentra quizá de manera más profunda, en la combinación de dicha situación con la legislación domestica que no tiene un enfoque integrado de derechos humanos en lo referente a la discapacidad, muy por el

\footnotetext{
${ }^{19}$ CRPD /C/QAT/1, párrafo 63.

${ }^{20}$ Al respecto, el Comité de Derechos Económicos, Sociales y Culturales de la ONU en su la Observación general No 20, La no discriminación y los derechos económicos, sociales y culturales (artículo 2, párrafo 2 del Pacto Internacional de Derechos Económicos, Sociales y Culturales), publicada el 2 de julio de 2009, destaca el hecho de que el mero tratamiento de la discriminación formal no garantizará la igualdad sustantiva. Este Comité subraya que eliminar la discriminación en la práctica requiere prestar suficiente atención a los grupos o individuos que sufren injusticias históricas o son víctimas de prejuicios persistentes. Véase $\mathrm{E} / \mathrm{C} .12 / \mathrm{GC} / 20$, párr. 8.

${ }^{21}$ El hecho de ratificar el Protocolo "promovería una implementación más efectiva de la CEDAW a través de la ampliación de su interpretación y de la aplicación práctica de la Convención (...) además podría crear una mayor conciencia pública sobre las garantías internacionales a los derechos humanos de las mujeres, así como una mayor atención a la CEDAW (...)". Véase FACIO, A. y OBANDO A.E., "Introducción sobre el proceso de elaboración del Protocolo Facultativo de la Convención sobre la Eliminación de Todas las Formas de Discriminación contra la Mujer", en Convención CEDAW y Protocolo Facultativo. Convención sobre la Eliminación de todas las formas de Discriminación contra la Mujer, Instituto Interamericano de Derechos Humanos, San José, Segunda edición, 2004, pp. 1516. Disponible en: https://www.iidh.ed.cr/IIDH/media/1838/convencicion-cedawprotocolof-2004.pdf (última consulta: 30 de mayo de 2017).
} 
contrario, siendo que ésta es concebida desde una perspectiva de modelo médico y no desde una perspectiva de modelo social.

\section{III.1.- Observaciones finales del Comité de la CDPD a Qatar respecto al Artículo 5 de la CDPD, relativo a la igualdad y no discriminación}

El Comité de la CDPD ha manifestado preocupación por el hecho de que el derecho qatarí no incluya una definición de ajustes razonables y que la denegación de este tipo de ajuste no se considere una forma de discriminación. "Le preocupa asimismo la falta de una legislación específica en materia de igualdad y no discriminación, incluido la protección contra la discriminación múltiple e interseccional." 22

El Comité de la CDPD le recomienda al Estado de Qatar que incorpore una prohibición explícita de la discriminación por motivo de discapacidad en su legislación nacional. También el Comité fecomienda a Qatar que incorpore en su legislación nacional una definición del concepto de ajustes razonables y que ésta sea aplicada de acuerdo con la Convención, en particular mediante el reconocimiento explícito de que la denegación de ajustes razonables constituye una discriminación por motivo de discapacidad. El Comité también recomienda al Estado parte que provea entrenamiento y formación sobre el concepto de ajustes razonables y sobre la no discriminación de las personas con discapacidad a las instituciones públicas y privadas, especialmente a quienes ejercen la profesión jurídica, la judicatura, los agentes del orden y a las propias personas con discapacidad. Por último, el Comité recomienda que sea recogida explícitamente en la legislación nacional la protección contra la discriminación múltiple e interseccional por motivo de género, edad, discapacidad y situación migratoria, entre otras causas, acompañada de sanciones más estrictas para los autores y una mayor indemnización y reparación para las víctimas. ${ }^{23}$

En cuanto al derecho a la igualdad de las personas con discapacidad y la obligación de realizar ajustes razonables, con carácter general vemos que dichos ajustes-adquieren su significado cuando el bien de la accesibilidad no se puede satisfacer de manera universal y se convierte así en un auténtico derecho destinado a remediar esa situación particular ${ }^{24}$.

De esta forma, el incumplimiento de la obligación de accesibilidad puede estar justificado o no, y ello depende de que se haya satisfecho o no el diseño universal (bien originariamente o bien

\footnotetext{
${ }^{22}$ CRPD/C/QAT/CO/1, párr. 11.

${ }^{23} \mathrm{CRPD} / \mathrm{C} / \mathrm{QAT} / \mathrm{CO} / 1$, párr. 12.

${ }^{24}$ Vid. PALACIOS, A., "El derecho a la igualdad de las personas con discapacidad y la obligación de realizar ajustes razonables", en I. Campoy (coord.), Los derechos de las personas con discapacidad: Perspectivas sociales, jurídicas y filosóficas, Dykinson, Madrid, 2004.
} 
a través de medidas de accesibilidad). Si se ha satisfecho, bien porque existe accesibilidad universal o bien porque no era posible 0 razonable que existiera, no podemos hablar de incumplimiento de la obligación de accesibilidad. Si no se ha satisfecho, porque no existe accesibilidad universal y era posible o razonable que existiera, podemos hablar de incumplimiento de la obligación de accesibilidad. Y en este segundo caso, estamos en presencia de una discriminación que debe arreglarse corrigiendo la situación y logrando la accesibilidad.

Un sentido integral de la accesibilidad implica: (i) el diseño universal, que funciona como un principio general fuente de obligaciones específicas; (ii) las medidas de accesibilidad, que aparecen cuando el diseño universal no se satisface; (iii) el ajuste razonable, que surgen cuando está justificado que la accesibilidad no sea universal.

Desde el eje de la accesibilidad, el sistema de derechos de las personas con discapacidad se proyecta en diferentes ámbitos entre los que destacan la salud, la libertad y seguridad, el empleo y trabajo, la capacidad jurídica, educación, el acceso a la justicia, la participación política, entre otros.

La accesibilidad se consigue mediante dos vías que no constituyen alternativas: en relación con los grupos y ex ante o en relación con los individuos. En relación con los grupos, la accesibilidad requiere del diseño universal, que implica que los bienes, espacios, servicios, procesos... sean utilizables por la mayor diversidad posible de personas sin necesidad de adaptación. La obligación de garantizar la accesibilidad en esta dimensión grupal subsiste a pesar de que en el origen no se haya diseñado para todos, y exige establecer objetivos y plazos para la eliminación de las barreras. A propósito de esta cuestión:

... Ios Estados parte deben fijar plazos y asignar recursos adecuados para la eliminación de las barreras existentes. Además, los Estados parte deben prescribir claramente los deberes que las diferentes autoridades (incluidas las regionales y locales) y entidades (incluidas las privadas) deben cumplir para asegurar la accesibilidad. Los Estados parte deben prescribir también mecanismos de supervisión efectivos que garanticen la accesibilidad y vigilen la aplicación de sanciones contra quienes incumplan las normas de accesibilidad. 25

En relación con los individuos, la estrategia para la accesibilidad son los ajustes razonables:

En el caso de las personas con deficiencias raras que no se tuvieron en cuenta al elaborar las normas de accesibilidad o que no utilizan los modos, métodos o medios previstos para garantizar la accesibilidad (no leen Braille, por ejemplo), incluso la aplicación de

25 Comité sobre los Derechos de las Personas con Discapacidad de la ONU, Observación general No 2 (2014), Articulo 9: Accesibilidad. CRPD/C/GC/2, párr. 24. 
las normas de accesibilidad puede ser insuficiente para garantizarles el acceso. En tales casos, pueden aplicarse ajustes razonables. ${ }^{26}$

En uno y otro caso, la accesibilidad es difícilmente clasificable en las categorías clásicas de los derechos (civiles, políticos o económicos, sociales y culturales) y afecta a los derechos de todas estas categorías por igual. Estas reflexiones pueden proyectarse sobre el análisis del reconocimiento del derecho a la salud, el derecho al trabajo, el derecho a la educación en Qatar.

En Qatar, las normas laborales por ejemplo, no mencionan conceptos como accesibilidad, ajustes razonables o adaptación del lugar de trabajo. En términos generales, la discapacidad no es lo suficientemente visible dentro del ámbito laboral en Qatar y las normas laborales deberían establecer explícitamente el principio de no discriminación y también deberían exigir la accesibilidad y adaptación del lugar de trabajo. Tal es el caso de la Ley No. 8 de 2009, sobre la Gestión de Recursos Humanos (que regula el empleo de los funcionarios públicos), en su Artículo 14, requiere que una persona nombrada en un puesto de trabajo pase las "pruebas, concursos y programas de calificación especificados por la agencia gubernamental correspondiente al puesto de trabajo", "debe tener las calificaciones y cumplir las condiciones especificadas para ese cargo", y "debe presentar un certificado médico de aptitud".

\section{IV.- A MODO DE CONCLUSIÓN}

Tras lo expuesto, resulta claro que la figura de los ajustes razonables no se encuentra dentro de la legislación qatarí. Esta situación de omisión se debe en gran parte a cómo se estructura la legislación qatarí respecto a las personas con discapacidad. En parte se debe también a que existen ciertos estereotipos y una visión generalizada respecto a los roles que las personas con discapacidad en Qatar deberían cumplir como meros pacientes.

Estos estereotipos son de corte proteccionista, coherente con la concepción de la discapacidad -como una condición de la persona- en el sistema jurídico qatarí, obedecen a un modelo de la discapacidad médico/rehabilitador y de corte caritativo. Es decir, estos estereotipos imposibilitan la consideración de las necesidades de las personas que están siendo estereotipadas, así como sus capacidades y deseos en base a intereses paternalistas. Lo cual, puede traducirse en falta de ajustes razonables en la información, lo que puede provocar un desempoderamiento de las personas con discapacidad.

El análisis que el Comité realiza al Estado de Qatar parte de la existencia de una discriminación hacia las personas con discapacidad desde el momento en que identifica la omisión de la figura de ajustes razonables. Al respecto, la Oficina del Alto Comisionado de las Naciones Unidas para los Derechos Humanos ha establecido en su

${ }^{26} \mathrm{CRPD} / \mathrm{C} / \mathrm{GC} / 2$, párr. 25. 
estudio temático sobre la Igualdad y no discriminación de acuerdo con el Artículo 5 de la Convención sobre los Derechos de las Personas con Discapacidad, lo siguiente:

Los ajustes razonables son una parte intrínseca del deber de no discriminación y, por tanto, se aplican a todos los derechos. En consecuencia, la denegación de ajustes razonables en relación con cualquier derecho constituye una discriminación por motivos de discapacidad. ${ }^{27}$

Para incluir la figura dentro del Estado de Qatar, es necesario comprender el concepto de ajustes razonables bajo el modelo social de la discapacidad. Qatar no es el único Estado que habiendo ratificado la CDPD haya omitido esta figura. Otros Estados también la omiten o confunden su concepto con el de medida de acción afirmativa o, incluso, con el concepto de accesibilidad.

Para una aplicación exitosa de los ajustes razonables, la Oficina del Alto Comisionado de las Naciones Unidas para los Derechos Humanos en el estudio temático ya mencionado, brinda ciertas clarificaciones que son de ayuda para los Estados que han ratificado la CDPD y que aún no han previsto en su legislación y en la práctica de sus sociedades, la figura de ajustes razonables. Naciones Unidas, a través de dicho estamento explica que es el Estado parte el que debe de proporcionar los elementos fundamentales para que los ajustes razonables se hagan posibles. El mismo Estado, a través de sus leyes nacionales, es también el que debe de tomar medidas para la realización de los ajustes razonables, o en su caso, obligar a los sectores privados (como empresas con trabajadores) para que las hagan posibles en lo que a éstas concierne. En particular, la Oficina establece que "los derechos, los deberes y los plazos de cada una de las partes implicadas deben estar claramente establecidos. Los Estados deben procurar elaborar directrices normativas, impartir formación específica y organizar actividades de sensibilización para difundir información pertinente a fin de promover actitudes y entornos inclusivos, y velar por que sus propios organismos y las entidades no estatales estén preparados para introducir ajustes razonables" 28 .

En definitiva, la situación jurídica respecto de la igualdad y no discriminación de las personas con discapacidad puede ser salvaguardada si el Estado toma en consideración la CDPD en su conjunto, como un todo. En definitiva, es clave la comprensión del concepto de ajustes razonables bajo la concepción del modelo social de discapacidad. Al mismo tiempo es fundamental en la aplicación,

\footnotetext{
${ }^{27}$ Igualdad y no discriminación de acuerdo con el artículo 5 de la Convención sobre los Derechos de las Personas con Discapacidad Informe de la Oficina del Alto Comisionado de las Naciones Unidas para los Derechos Humanos, 9 de diciembre de 2016. A /HRC/34/26, párr. 27.

${ }^{28}$ A /HRC/34/26, párr. 39.
} 
distinguir entre políticas públicas y legislaciones en materia de accesibilidad y las que se refieren a ajustes razonables.

La propia CDPD y su Comité nos dan a entender que los ajustes razonables deberían estar al alcance de todas las personas, ya que la diversidad es lo que nos caracteriza. Este alcance tiene que estar bajo el amparo de un modelo social de discapacidad y no bajo un modelo de caridad de la discapacidad. 